\title{
Millimeter-wave radar micro-Doppler feature extraction of consumer drones and birds for target discrimination
}

Samiur Rahman, Duncan A. Robertson

Samiur Rahman, Duncan A. Robertson, "Millimeter-wave radar micro-Doppler feature extraction of consumer drones and birds for target discrimination ," Proc. SPIE 11003, Radar Sensor Technology XXIII, 110030S (3 May 2019); doi: $10.1117 / 12.2518846$

Event: SPIE Defense + Commercial Sensing, 2019, Baltimore, Maryland, United States 


\title{
Millimeter-wave radar micro-Doppler feature extraction of consumer drones and birds for target discrimination
}

\author{
Samiur Rahman ${ }^{\mathrm{a}}$, Duncan A. Robertson ${ }^{\mathrm{a}}$ \\ ${ }^{a}$ University of St Andrews, SUPA School of Physics \& Astronomy, St Andrews, Fife KY16 9SS, \\ Scotland
}

\begin{abstract}
This paper discusses the various millimeter-wave radar micro-Doppler features of consumer drones and birds which can be fed to a classifier for target discrimination. The proposed feature extraction methods have been developed by considering the micro-Doppler signature characteristics of in-flight targets obtained with a frequency modulated continuous wave (FMCW) radar. Three different drones (DJI Phantom 3 Standard, DJI Inspire 1 and DJI S900) and four birds of different sizes (Northern Hawk Owl, Harris Hawk, Indian Eagle Owl and Tawny Eagle) have been used for the feature extraction and classification. The data for all the targets was obtained with a fixed beam W-band $(94 \mathrm{GHz})$ FMCW radar. The extracted features have been fed to two different classifiers for training (linear discriminant and support vector machine (SVM)). It is shown that the classifiers using these features can clearly distinguish between a drone and a bird with $100 \%$ prediction accuracy and are able to differentiate between various sizes of drones with more than $90 \%$ accuracy. The results demonstrate that the proposed algorithm is a very suitable candidate as an automatic target recognition technique for a practical FMCW radar based drone detection system.
\end{abstract}

Keywords: Micro-Doppler, Radar, FMCW, Millimeter-wave, classification, drones, birds, support vector machine, linear discriminant

\section{INTRODUCTION}

Rotary wing drones and birds produce distinctive micro-Doppler signatures in their radar signal returns ${ }^{1,2}$. These signatures can be used for target discrimination, which is a very critical feature for a drone surveillance radar. As these targets have relatively low bulk radar cross section (RCS) ${ }^{3}$ of around $-20 \mathrm{dBsm}$, with the propeller RCS being 20-30 dB below that, obtaining high fidelity micro-Doppler signatures is not always guaranteed. To increase micro-Doppler sensitivity, millimeter-wave radar can be used which offers larger micro-Doppler spread and better Doppler resolution for a given integration time than lower frequency $\operatorname{radar}^{4,5}$. Still, a robust feature extraction method is needed for accurate classification due to the presence of clutter in the signal as the targets may fly with relatively low altitude.

In recent years, a number of research works have proposed different feature extraction methods for drone and bird classification $^{6-8}$. The classification algorithms in those reports are mainly based on micro-Doppler, along with bulk Doppler shift and target RCS. The feature extraction methods usually use singular value decomposition (SVD) to extract the frequency and time axis information from a spectrogram separately. $\mathrm{In}^{7}$, Doppler bandwidth and centroid based feature extraction is reported, illustrating more than $90 \%$ accurate classification, mainly for hovering targets. $\mathrm{In}^{6}$, alignment of bulk velocity is performed but the overall classification process is developed for continuous wave (CW) radar data, which has a higher Doppler sampling rate hence can unambiguously sample the fast rotation of drone propeller blades, unlike FMCW radar. However, it is vital to consider the micro-Doppler signatures using FMCW radar data, as FMCW radar is a more realistic candidate for drone detection radar sensor, due to its ability to locate targets.

In this paper, we propose an automated target recognition system addressing the issues mentioned above and based on staring mode FMCW radar. Even though a scanning radar covers more area, a fixed beam system can provide very highfidelity classification performance by utilizing longer Doppler integration. As the RCS of drones and birds are comparable $^{3}$, the feature extraction approach is entirely micro-Doppler based. Having said that, RCS values are used in the extraction of one of the micro-Doppler features. It will be shown that even though RCS alone cannot be a reliable feature, absolute RCS values obtained from a well-calibrated radar can be useful to filter out clutter from micro-Doppler components within the spectrogram. The whole feature extraction method is described in detail in this paper. The method is then used on real data obtained from different types of drones and birds. Finally, the high accuracy performance of the features used for classification is demonstrated.

Radar Sensor Technology XXIII, edited by Kenneth I. Ranney, Armin Doerry, Proc. of SPIE Vol. 11003, 110030S · (c) 2019 SPIE · CCC code: 0277-786X/19/\$18 · doi: 10.1117/12.2518846 


\section{PROPOSED MICRO-DOPPLER BASED FEATURE EXTRACTION}

For the proposed method, spectrograms obtained by Short Time Fourier Transform (STFT) are used, as these are easily produced and visually intuitive. The first step is the Doppler alignment after which the following four features are extracted from the spectrogram plots:-

- Micro-Doppler spread (width)

- Micro-Doppler spread (weight)

- Micro-Doppler strength

- Micro-Doppler periodicity

\subsection{Doppler alignment}

As the bulk velocity of drones and birds can be quite similar ${ }^{9}$, the strong bulk Doppler signature becomes redundant from the feature extraction point of view. Also, the presence of strong zero Doppler (corresponding to clutter and/or hovering targets) can be removed as our focus is only on the micro-Doppler. To enhance the micro-Doppler components, the bulk Doppler is aligned with the zero Doppler. To achieve that, zero Doppler suppression is done first. Then for each time slice of the spectrogram, the maximum signal location is found which will typically correspond to the bulk Doppler. The whole time slice is then circular shifted according to the measured offset of the bulk from the zero Doppler. To filter out further clutter, the dynamic range of the spectrogram is reduced to eliminate low level signals. It is known that the microDoppler is typically around $20-30 \mathrm{~dB}$ lower than the bulk but to provide some margin we have chosen to limit the dynamic range to $60 \mathrm{~dB}$ below the maximum value. Fig. 1 shows example $94 \mathrm{GHz}$ spectrograms for a drone and a bird before and after zero Doppler suppression and bulk Doppler alignment, with a dynamic range of $60 \mathrm{~dB}$.
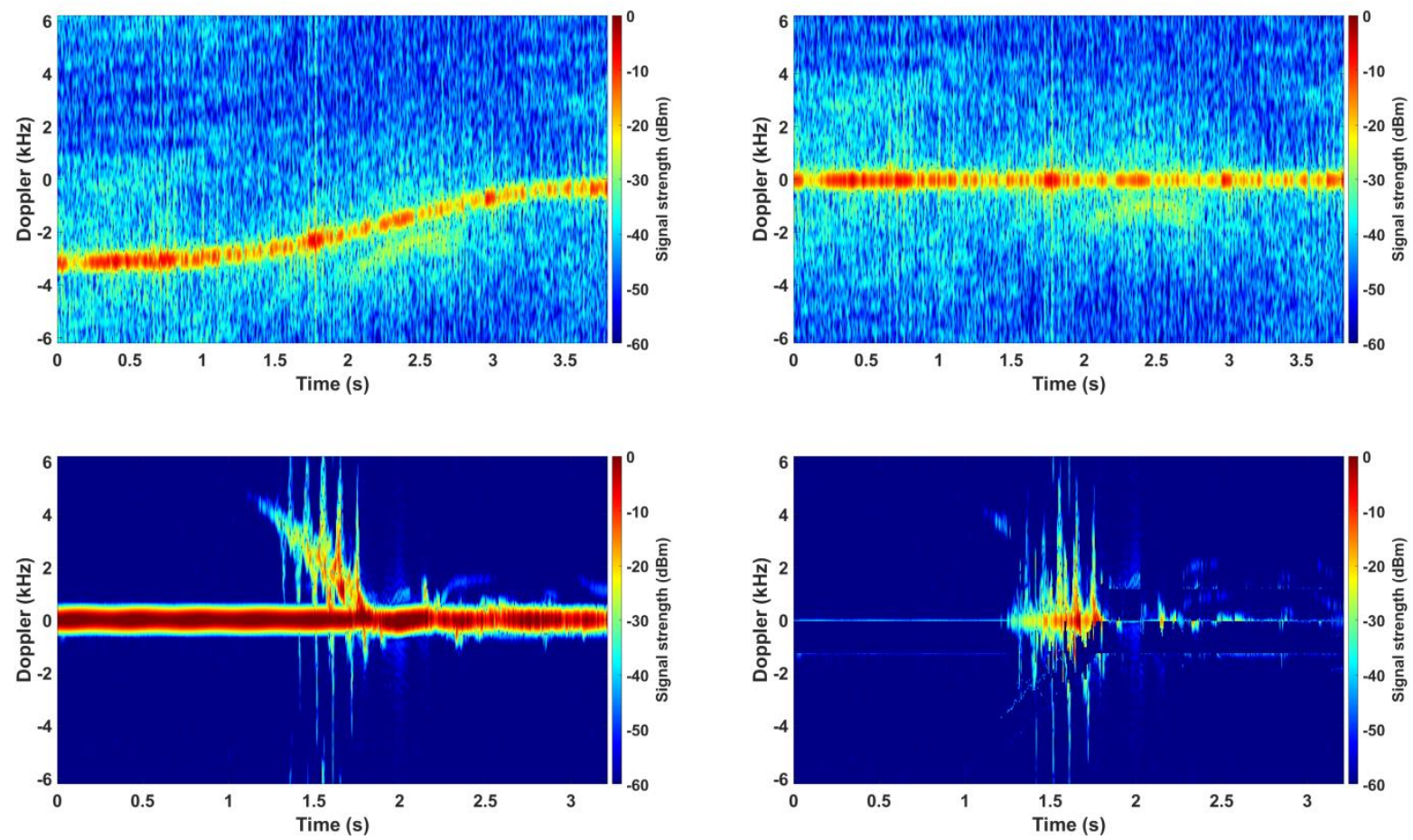

Figure 1. Example $94 \mathrm{GHz}$ spectrograms of a DJI Inspire (top) and a Northern Hawk Owl (bottom) showing the effect of zero Doppler suppression and bulk doppler alignment. Images on the left show the spectrograms without these compensations and on the right show after the processes have been applied.

This Doppler alignment makes the feature extraction procedure much easier, as anything beyond the zero Doppler can be regarded as micro-Doppler return. Also, it allows one to calculate the Doppler periodicity more accurately as a Doppler slice from the spectrogram now mainly consists of drone blade flashes or bird wing beats (if present). 


\subsection{Feature extraction}

The first three features mainly correspond to the Doppler axis of the spectrogram, whereas the last one corresponds to the time axis. The idea is to quantify the extent of the micro-Doppler frequency content and its change with time.

\subsubsection{Micro Doppler Spread (Width)}

To calculate the weighted spread of the micro-Doppler information in a spectrogram, a widely used decomposition method like SVD can be used ${ }^{6}$. The problem with conventional SVD is that a reliable micro-Doppler spread can only be obtained if the dynamic range of the spectrogram is quite large, excluding the bulk component. This means, the microDoppler signal-to-noise ratio needs to be quite high. In practice, the micro-Doppler signal strength from the plastic blade of a commercial drone may be very small and close to the noise floor. This means that the values obtained from SVD corresponding to the micro-Doppler are very small, and hence it is hard to define a clear threshold to separate them from noise. For this reason, we proposed an algorithm which calculates the spread based on the number of micro-Doppler occupancies within a spectrogram. This feature extraction process creates a one-dimensional array of the Doppler axis in which each array element value quantifies the presence of a micro-Doppler signal above a given threshold. The threshold value is a hard threshold set to be some $\mathrm{dB}$ above the noise floor to avoid false counts. The calculation is performed as:-

$$
\text { Swidth }(i)=\frac{\text { Number of elements above threshold in a single time slice }}{\text { Total number of elements in a single time slice }}
$$

Here, Swidth(i) is the micro-Doppler spectral width for the ith Doppler point of the spectrogram, which is normalized to maximum value of 1 . It should be noted that using the SVD method can also provide this spread information (from the decomposed matrix corresponding to the Doppler axis), but not with high accuracy due to the low signal return from the propellers. Fig. 2 compares micro-Doppler spread plots for a drone and a bird obtained with conventional SVD and the method proposed above (using a threshold of $-50 \mathrm{dBm}$ ).
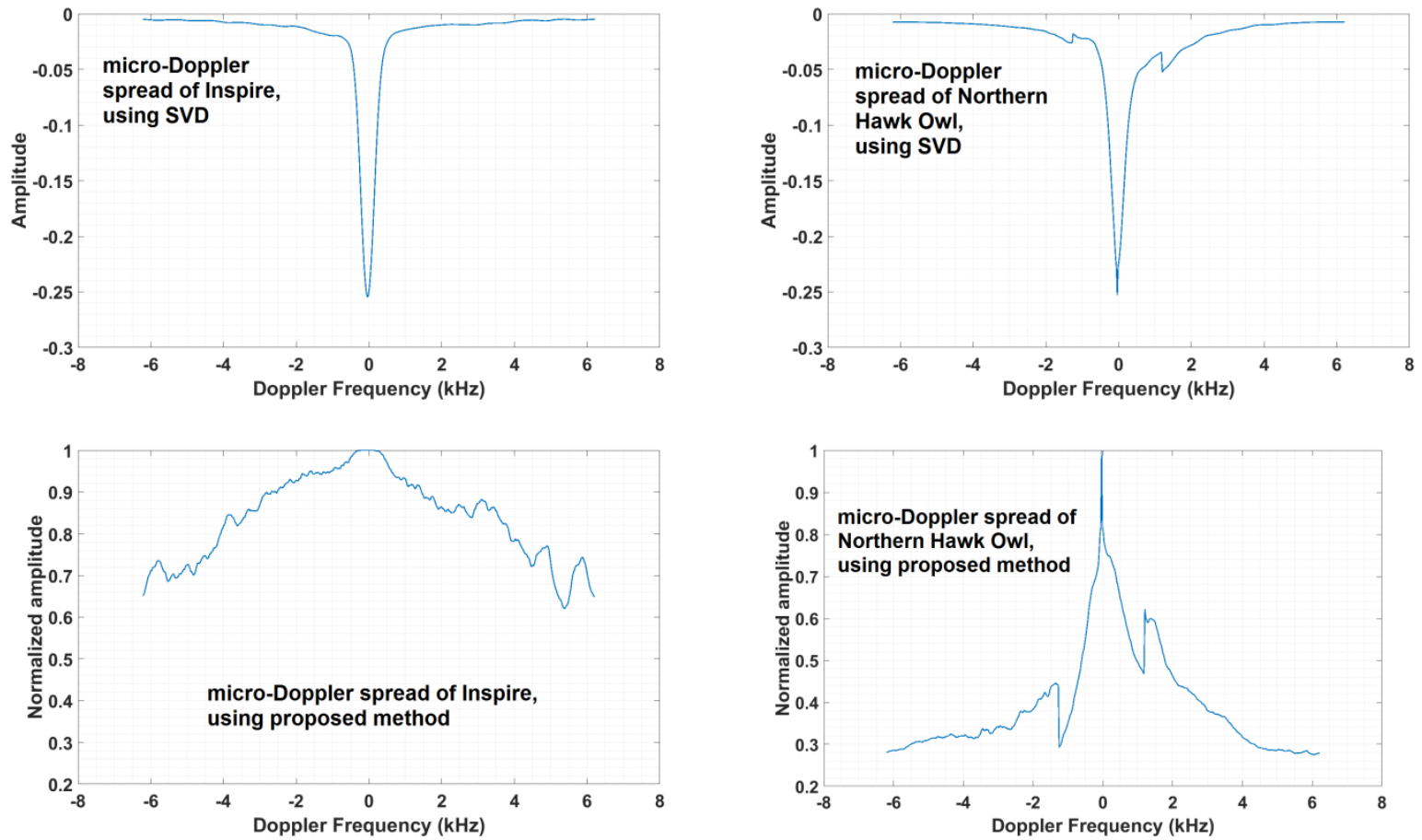

Figure 2. Example of micro-Doppler spread plots. The top left one shows the spread plot of the Inspire obtained by SVD matrix and the top right is the same plot for the Northern Hawk Owl. The bottom left is obtained by using the proposed method showing larger spread. The bottom right is the same plot obtained for the Northern Hawk Owl

It can be seen from Fig. 2 that the proposed method performs better than the conventional SVD. The variation between the plots for the Inspire and the Northern Hawk Owl obtained with SVD is minimal. In contrast, the difference in spread can be easily visualized from the plots obtained by the proposed method. For the classifier feed, a single value for the 
whole spectrogram is required. It is obtained by having another threshold on the plots and simply calculating the number of data points above the threshold. From Fig. 2 (lower plots), if the threshold is set to 0.5, all the data points are then above the threshold for the Inspire which is 512 points in this case. For the Northern Hawk Owl, the value is 86 . The difference confirms the validity of the feature in a FMCW radar spectrogram, since the micro-Doppler spread for a drone is usually quite large compared to that obtained from birds.

\subsubsection{Micro-Doppler Spread (Weight)}

From Fig. 2 (lower plots), it is also observed that most of the individual data point values are lower for the bird than the drone. This is expected since when taking a time slice from a bird spectrogram there are fewer points without any Doppler information, hence a lower total value. This property can also be used as a feature and can be very easily extracted from the plots. This is achieved simply by calculating the area under the micro-Doppler spread (width) curve to give a single value corresponding to the micro-Doppler spread weight, Sweight $=\sum_{i=1}^{M} \operatorname{Swidth}(i)$, where $M$ is the number of data points in the Doppler axis.

\subsubsection{Micro-Doppler Strength}

This feature calculates the overall strength of the micro-Doppler in the spectrogram. The preceding two features mainly measure the number of occurrences of the micro-Doppler signal in a spectrogram. On the other hand, this feature measures the micro-Doppler intensity in terms of the received signal power. It should be noted that this feature can only be reliably used when absolute RCS values are available. This is because to calculate micro-Doppler strength, a range scaling factor must be used for spectrograms collected at different ranges. The straightforward scaling factor is to convert the received power to the corresponding RCS value, which requires the radar to be calibrated. As it is quite trivial to plot the predicted radar return from different ranges for a given RCS if the link budget of the radar system is known, this criterion is not a limiting factor. Ultimately, it increases the feature reliability, hence improving the classification performance. For a spectrogram obtained from multiple range bins, the median range value can be selected which is a good approximation for determining the final value. We define the single value for the micro-Doppler strength as Dstrength $=\sum_{i=1}^{M} \sum_{j=1}^{N} S(i, j)$, where $S(i, j)$ is the signal strength of the ith element of the Doppler axis and $j$ th element of the time axis. $M$ and $N$ are the total number of data points on the Doppler and time axes, respectively. In practice, the elements corresponding to the zero Doppler and its close vicinity are omitted, to exclude the contributions from the bulk.

\subsubsection{Micro-Doppler Periodicity}

It is very hard to extract the rotor rotation rate of a drone from FMCW radar data due to the hardware constraints of requiring a very high chirp repetition frequency (CRF) in order to sample the Doppler at a sufficiently high rate. Also, such high CRF increases the computational load as more data points then need to be processed. Nonetheless, wing beat rates of birds can be extracted easily from FMCW data with practical CRFs which then can be a very useful feature to discriminate the drone from the bird. The unitary matrix corresponding to the time axis obtained from SVD is used in this case. From the matrix, the first row vector is then selected as this contains the maximum information almost all the time. This vector can be regarded as a one-dimensional representation of the periodicity observed in the spectrogram (i.e. bird wing beats). As the vector is still in the time domain, Fourier transformation of this is then performed and the first peak is then selected as the single value for the classifier.

For better illustration of the process, $94 \mathrm{GHz}$ continuous wave $(\mathrm{CW})$ radar data of a tethered quadcopter (DJI Phantom 3 Standard) with only one propeller is used. The sampling rate was $100 \mathrm{kHz}$ to ensure unambiguous sampling of the Doppler frequency. The rotor blade flashes are now clearly observed in the spectrogram plot shown in Fig. 3. The time difference between the two peaks is measured to be $0.011 \mathrm{~s}$, corresponding to $90.91 \mathrm{~Hz}$. As can be seen from the frequency spectrum plot at the top right of Fig. 3, the periodicity is measured as $90.01 \mathrm{~Hz}$, in very close agreement with the time domain value. This value of the fundamental frequency is then directly fed to the classifier. It should be noted that as the rotor has two blades, the actual rotation rate of the rotor is $90.91 / 2=45.45 \mathrm{~Hz}$. The bottom left plot is an equivalent one for a DJI Inspire 1 derived from the FMCW spectrogram (12.4 kHz CRF) in Fig. 1 and the lack of distinct periodic features is evident. The bottom right plot is the corresponding one obtained for the Northern Hawk Owl FMCW spectrogram in Fig. 1. From that image, the wing beat rate can be roughly determined to be $9-10 \mathrm{~Hz}$. Again, it is seen that the processed value $(8.698 \mathrm{~Hz})$ is very close to the time domain value. 

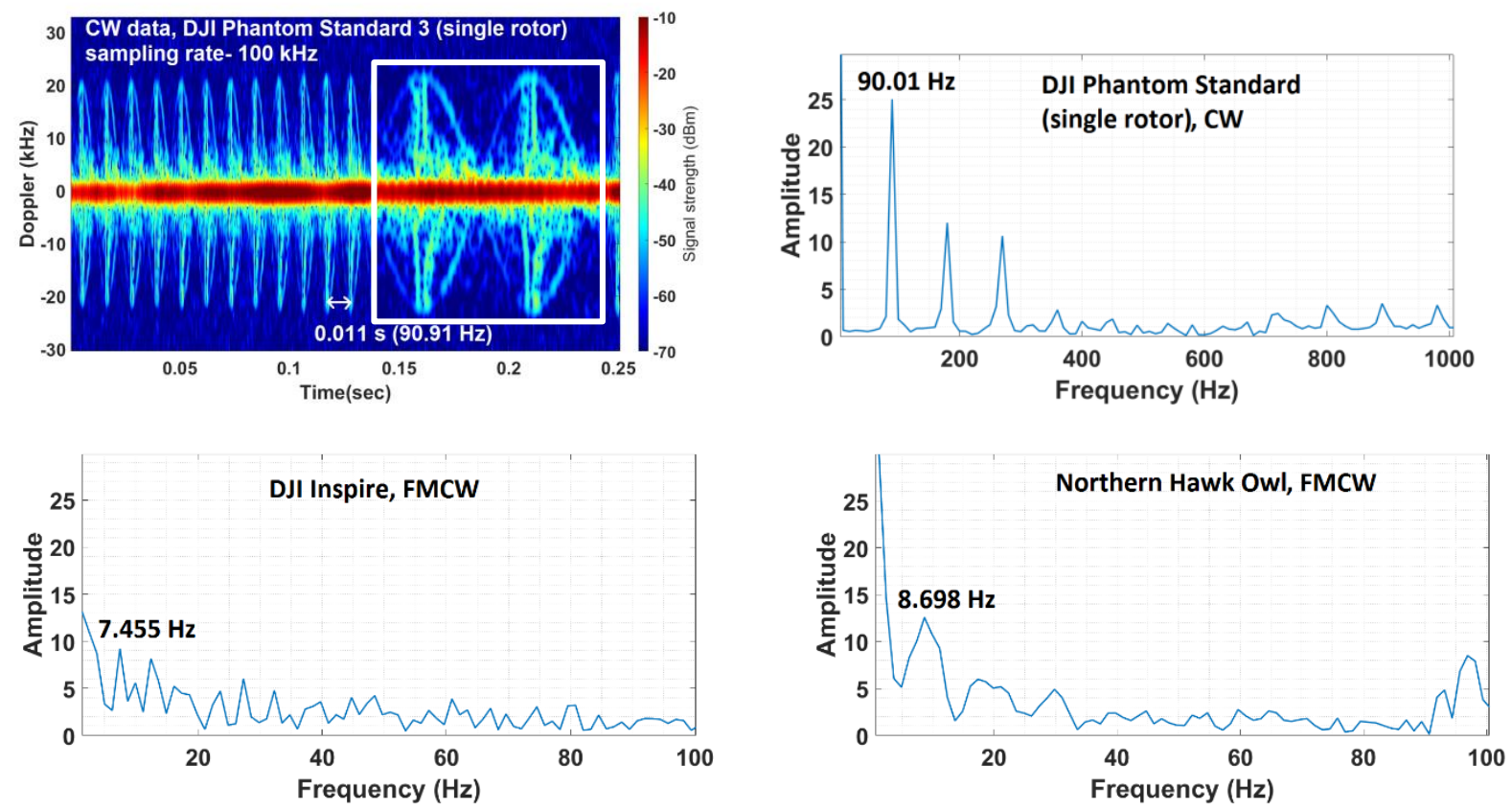

Figure 3. Example of micro-Doppler periodicity calculation. The $94 \mathrm{GHz} \mathrm{CW}$ spectrogram at the top left illustrates the rotor blade flashes of a single rotor of a tethered DJI Phantom Standard 3; the inset plot reveals the full detail of two flashes when magnified. Top right plot shows the rotor blade flash frequency is obtained from this CW spectrogram by Fourier transforming the first row vector of the SVD unitary matrix, providing the periodicity value for the classifier. Bottom left plot shows the periodicity value obtained for DJI Inspire FMCW spectrogram shown in Fig. 1. Bottom right is the corresponding plot obtained for the Northern Hawk Owl FMCW spectrogram shown in Fig. 1.

One problem with this feature extraction method for FMCW radar drone data is that it produces some unexpected low level peaks at low frequencies on the periodicity plot. One might expect that the periodicity value would be zero as there is no significant periodicity present in the spectrogram (as seen in Inspire spectrogram in Fig. 1) since the Doppler spectrum was under-sampled and the propellers rotate at slightly different speeds. It would be risky to filter the low frequency components out as they are often comparable to bird wing beat frequencies. Instead, adjusting the amplitude threshold for first peak detection can be used to minimize this problem. As can be seen in Fig. 3, if the threshold is set to 10, the periodicity value for the Northern Hawk Owl is $8.698 \mathrm{~Hz}$ and for the DJI Inspire it becomes zero (the large DC response is discarded). Still, there is no guarantee that this threshold will consistently reject frequencies corresponding to drones while allowing the ones that relate to birds. Nonetheless, this feature is still very useful as it provides actual bird wing beat frequency values from FMCW spectrogram data and actual drone propeller rotation rates from $\mathrm{CW}$ spectrogram data. It could obtain propeller rotation rate from FMCW data, but that would require a very high CRF.

\section{TARGET DISCRIMINATION RESULTS}

The relevant details of the targets used as classes are given below-

Drones:

- DJI Phantom Standard 3 (weight $1.216 \mathrm{~kg}$, width $35 \mathrm{~cm}$, blade length $13 \mathrm{~cm}$ )

- DJI Inspire 1 (weight $2.845 \mathrm{~kg}$, width $58 \mathrm{~cm}$, blade length $34.5 \mathrm{~cm}$ )

- DJI S900 (weight $3.3 \mathrm{~kg}$, width $90 \mathrm{~cm}$, blade length $38.1 \mathrm{~cm}$ )

Birds:

- Northern Hawk Owl (weight $0.26 \mathrm{~kg}$, length $40 \mathrm{~cm}$, wingspan $45 \mathrm{~cm}$ )

- Harris Hawk (weight $0.71 \mathrm{~kg}$, length $55 \mathrm{~cm}$, wingspan $115 \mathrm{~cm}$ )

- Indian Eagle $O w l$ (weight $0.97 \mathrm{~kg}$, length $52 \mathrm{~cm}$, wingspan $135 \mathrm{~cm}$ )

- Tawny Eagle (weight $1.84 \mathrm{~kg}$, length $65 \mathrm{~cm}$, wingspan $175 \mathrm{~cm}$ ) 
All the data was collected using a coherent $94 \mathrm{GHz}$ FMCW radar named T-220, which has a very low phase noise ${ }^{10}$. The radar has a homodyne architecture with dual fan beam antenna $\left(0.9^{\circ}\right.$ azimuth and $3^{\circ}$ elevation beamwidths, $40.5 \mathrm{dBi}$ gain, circular polarization) and with transmit power of $+18 \mathrm{dBm}$. The chirp period is set at $80.5 \mu \mathrm{s}$ with a CRF of 12.4 $\mathrm{kHz}$ so the maximum unambiguous velocity range is $\pm 9.93 \mathrm{~ms}^{-1}$. It should be mentioned that the $\mathrm{CW}$ result shown in Fig. 3 is also produced by running the T-220 radar in CW mode.

A total of 28 spectrogram plots (4 for each class) have been processed to extract the four feature values. The dwell time varied between $3-3.5 \mathrm{~s}$, long enough for good Doppler integration but not too long to degrade the classification update rate. STFT operation is performed to obtain the spectrograms with the sliding window length being 512 samples (41.2 ms). A Gaussian window (with $\alpha=4$, where $\alpha$ is inversely proportional to the standard deviation) is used for the optimum simultaneous time and frequency resolution, a variant of STFT also known as the Gabor transform ${ }^{2}$. The window sliding is done with $95 \%$ overlap.

The Classification Learner App in MATLAB ${ }^{\circledast}$ was used to feed the data to the classifier and to train the model. The Linear Discriminant and SVM classifiers were chosen to test the reliability of the features, as both are very widely used. All four features described above (micro-Doppler spread (width), micro-Doppler spread (weight), micro-Doppler strength and micro-Doppler periodicity) are used in each case. At first, the classification training results are analyzed for only two classes, where all the drones are of one class (Drone) and all the birds are part of the other (Bird). Then, the training is performed by having all the targets as individual classes to observe the capability of distinguishing between different types of drones and birds. During training and confusion matrix generation, 5-fold cross validation is used all the time.

Fig. 4 shows a screenshot taken during the training of the classifiers. It is seen that both Linear Discriminant and SVM have successfully predicted the classes with $100 \%$ accuracy. The example scatter plot seen in the figure provides a visual example of the target separation showing only the micro-Doppler spread (width) and micro-Doppler strength values for all the classes. The blue dots representing the birds are clustered together and are clearly separated from the red dots (drones). This demonstrates that the proposed method can be used to discriminate between a drone and a bird with very high level of confidence.

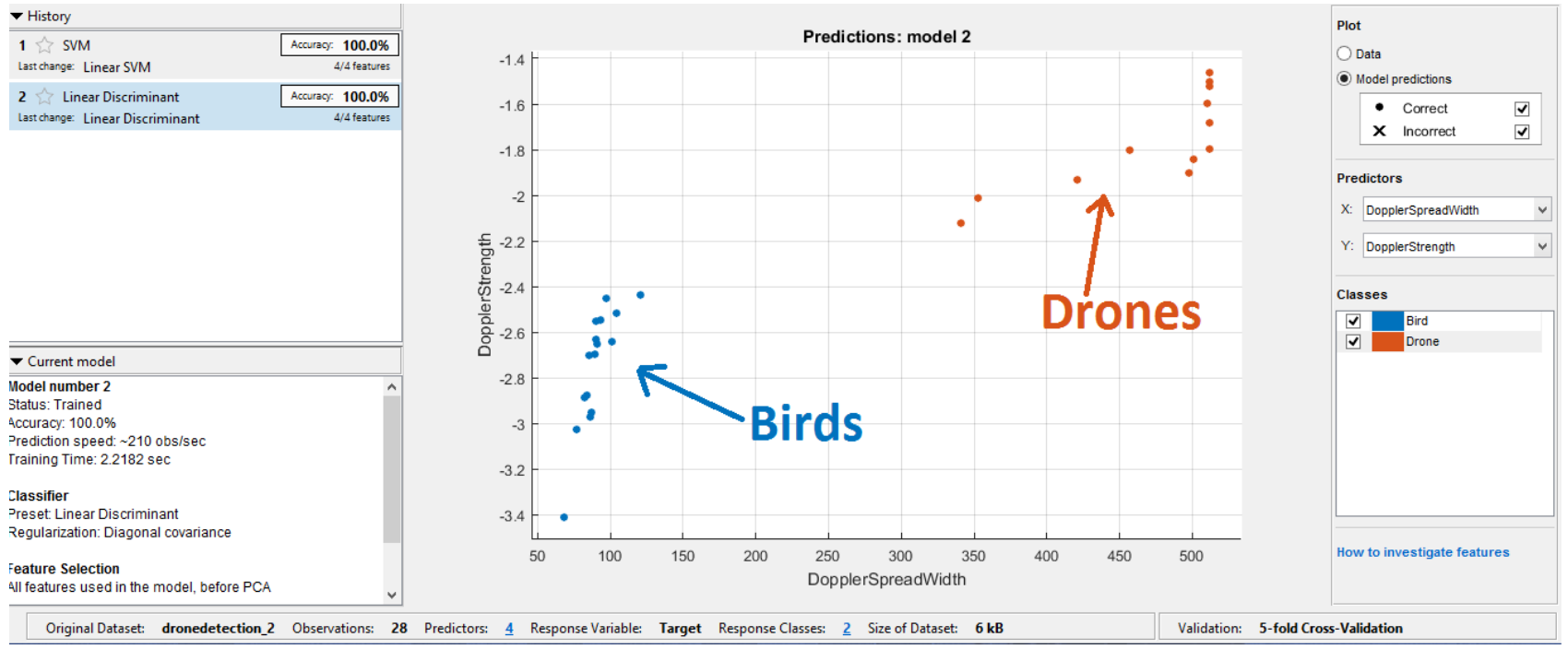

Figure 4. Screenshot of the Classification Learner App in MATLAB while performing the training for 2 classes, showing $100 \%$ accuracy with both classifiers

Next, the classifiers are trained only with the drone data. As seen in Fig. 5, the prediction accuracy for the Linear Discriminant is $100 \%$ whereas for SVM, it is $91.7 \%$. This shows that the target discrimination algorithm has the potential to separate between various types of drones. From the scatter plot, it is observed that the Phantom and the S900 are quite widely spaced and the Inspire feature values are roughly in the middle. This separation is directly correlated to the micro-Doppler signal return, which is much higher for the S900 than the Phantom, corresponding to larger values for all the features except periodicity. 

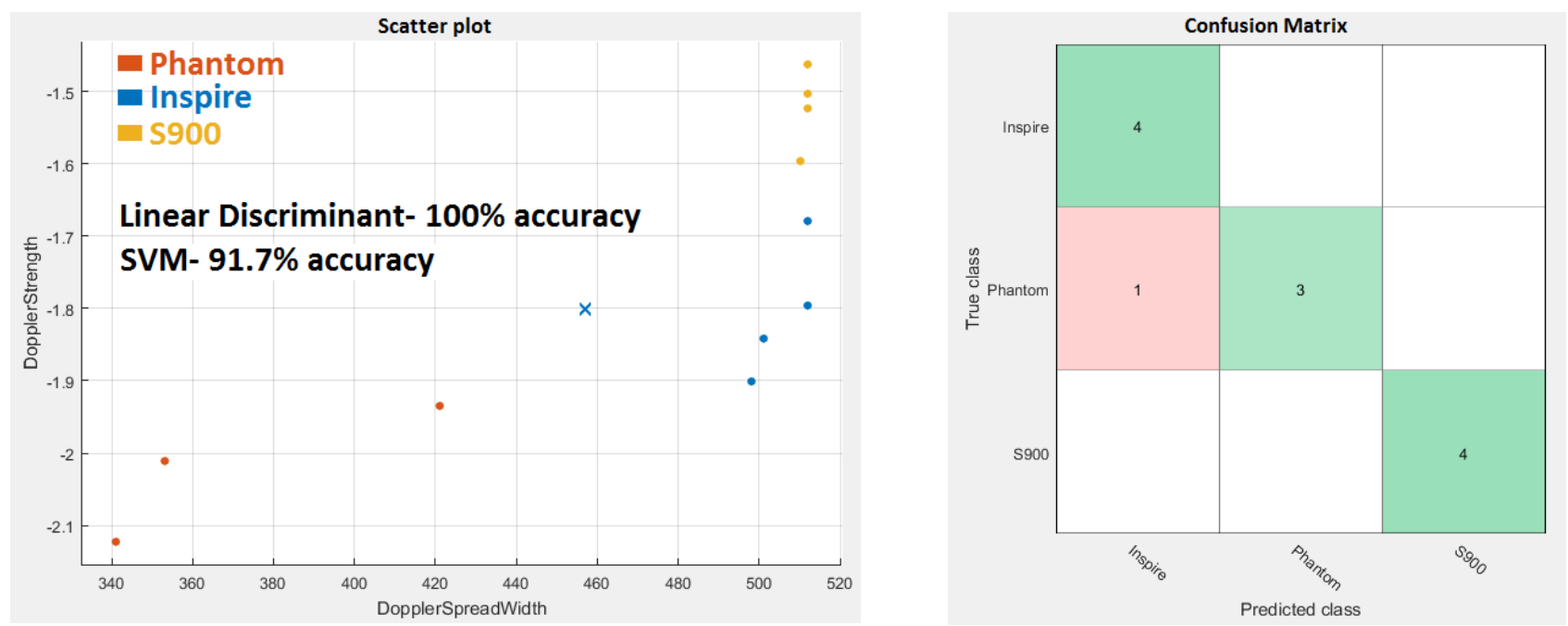

Figure 5. Scatter plot (left) generated by SVM for 3 classes corresponding to three types of drones, showing the target distribution in terms of micro-Doppler spread (width) and micro-Doppler strength. Confusion matrix (right) is generated after training the classifier with all four features

On the other hand, when trained with all four classes of bird, the classifiers are not at all able to distinguish between them. From the example scatter plot in Fig. 6, the reason can be understood: the micro-Doppler periodicity values of the four birds are too similar for the classifiers to isolate.
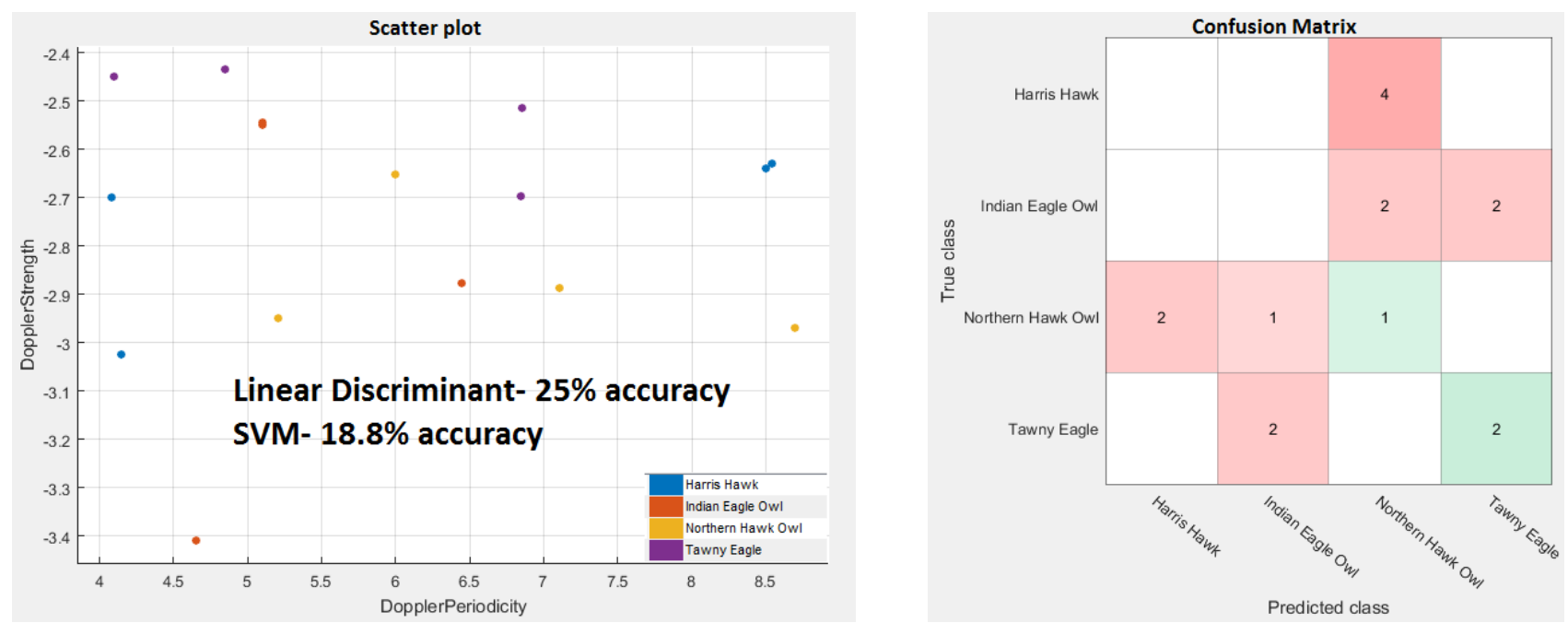

Figure 6. Scatter plot (left) generated by SVM for 4 classes corresponding to four types of birds, showing the target distribution in terms of micro-Doppler strength and micro-Doppler periodicity. Confusion matrix (right) is generated after training the classifier with all four features

In this case, to discriminate effectively, the physical sizes of the birds would need to be significantly different which would create more variation in the other three features. To test this hypothesis, classifiers were trained only with the smallest bird (Northern Hawk Owl) and the largest bird (Tawny Eagle). Fig. 7 shows that in this case, the Linear Discriminant is able to predict with $87.5 \%$ accuracy, whereas SVM predicts with $100 \%$ accuracy. This confirms the idea that Doppler strength and Doppler spread (weight) are related to bird size and may be used to classify different types of birds. 

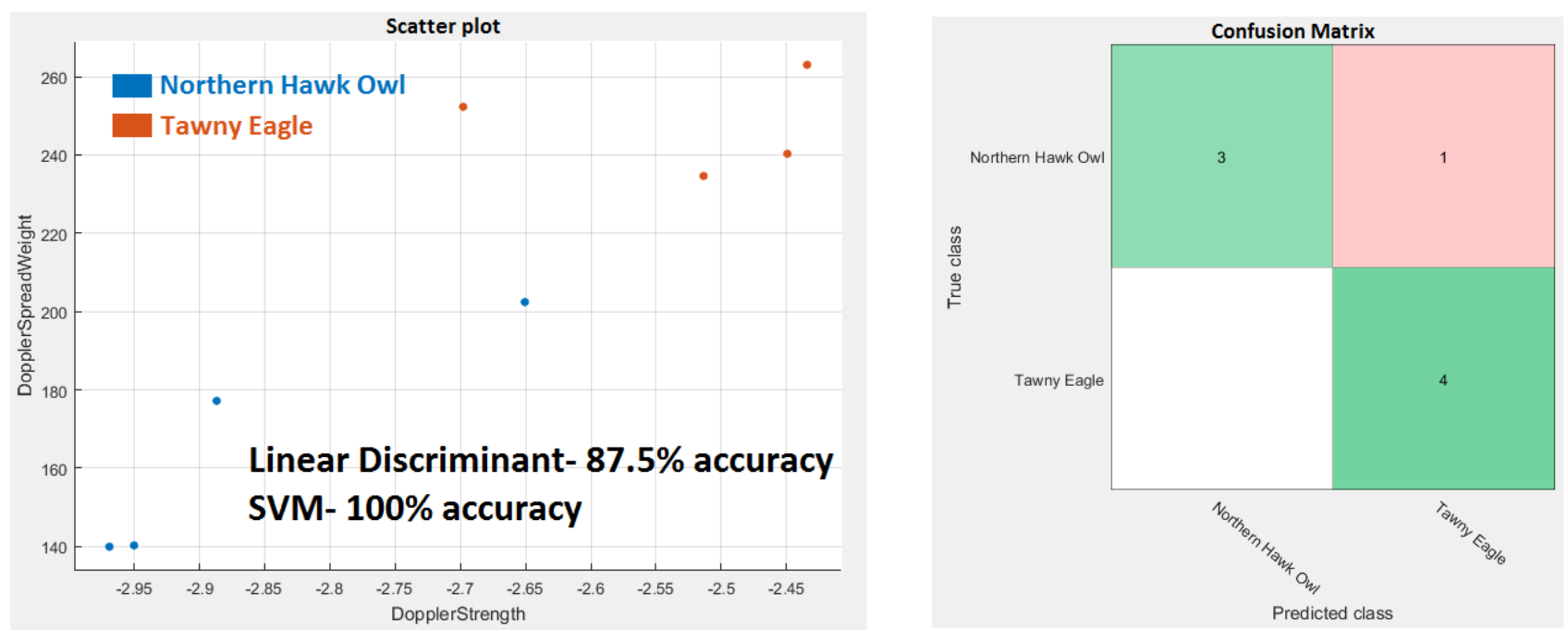

Figure 7. Scatter plot (left) generated by linear discriminant for 2 classes corresponding to the Northern Hawk Owl and the Tawny Eagle, showing the target distribution in terms of micro-Doppler spread (weight) and micro-Doppler strength. Confusion matrix (right) is generated after training the classifier with all four features

From all the combinations of scatter plot shown above, it has been observed that the micro-Doppler spread (weight \& width) and the micro-Doppler strength are the dominant features that improve the accuracy of classification as selection of these two features provide the best separation in the scatter plots. This again points to the importance of high microDoppler sensitivity in the radar system for achieving better classification. For a given sampling rate, higher operating frequencies will produce a higher Doppler frequency and increasing the Doppler frequency range improves the chance of greater feature separation.

\section{CONCLUSION AND FUTURE WORKS}

The main objective of this study was to develop an algorithm for automatic drone classification in realistic in-flight scenarios, with a practical sensor such as an FMCW radar. As birds are the main confusers, the ability to distinguish between a drone and a bird was the primary requirement. Four different micro-Doppler based feature extraction methods have been discussed. The proposed discrimination method also incorporates pre modification of the data by aligning the spectrogram plot with respect to zero Doppler. The method is then used on real data obtained for three different drones and four different birds. To verify the performance, the feature values are then used to train two well-known classifiers, Linear Discriminant and SVM. It was clearly shown that the features are very distinctive, enabling identification of the targets, with both the classifiers showing $100 \%$ prediction accuracy during training. It was also shown that the proposed method can recognize different types of drones, especially when they vary in size. More than $90 \%$ accuracy was obtained by both the classifiers. Much lower accuracy was observed with the birds as their features overlapped with each other when all four birds were considered. However, the prediction performance improved significantly by picking two birds of significantly different size, while omitting the other two. Overall, it has been demonstrated that the proposed algorithm has very good potential to be used in a drone detection FMCW radar system, to classify drones and birds in a dynamic environment. The method described in this paper uses thresholds very close to the noise floor to extract the micro-Doppler information which requires high fidelity Doppler measurements, best achieved by using a very low phase noise radar with excellent spectral purity.

In the future, the intention is to put the algorithm to more rigorous test by increasing the dataset for the classifier training. This can be achieved by processing more data relating to the classes used in this paper for feature extraction. Also, new classes can be added by taking data from other types of drones and birds to expand the database. Finally, data from other locations with surrounding clutter different from the current one will be quite useful. This will provide the opportunity to optimize the feature extraction algorithms as they use threshold values at various points. 


\section{ACKNOWLEDGEMENTS}

The authors acknowledge the funding received from the Science and Technology Facilities Council which has supported this work under grant ST/N006569/1. The authors acknowledge and thank Dr Robert I. Hunter, Dr Adeola Fabola and Dr Paddy Pomeroy for flying the drones. The authors also thank Elite Falconry for flying the birds.

\section{REFERENCES}

[1] Chen, V. C., [The micro-doppler effect in radar], Artech House (2011).

[2] Chen, V. C., Li, F., Ho, S.-S. and Wechsler, H., “Analysis of micro-Doppler signatures,” IEE Proc. - Radar, Sonar Navig. 150(4), 271 (2003).

[3] Rahman, S. and Robertson, D. A., "In-flight RCS measurements of drones and birds at K-band and W-band," IET Radar, Sonar Navig. (2018).

[4] Rahman, S. and Robertson, D. A., "Millimeter-wave micro-Doppler measurements of small UAVs," Proc. SPIE 10188, Radar Sens. Technol. XXI 10188, K. I. Ranney and A. Doerry, Eds., 101880T, International Society for Optics and Photonics (2017).

[5] Rohling, H. (Hermann), Technische Universität Hamburg-Harburg. and Deutsche Gesellschaft für Ortung und Navigation., "Millimeter-wave radar micro-Doppler signatures of human motion," Int. Radar Symp. IRS 2011, DCM Druck Center, Leipzig (2011).

[6] Molchanov, P., Harmanny, R. I. A., de Wit, J. J. M., Egiazarian, K. and Astola, J., "Classification of small UAVs and birds by micro-Doppler signatures,” Int. J. Microw. Wirel. Technol. 6(3-4), 435-444 (2014).

[7] Ritchie, M., Fioranelli, F., Borrion, H. and Griffiths, H., "Classification of loaded/unloaded micro-drones using multistatic radar," Electron. Lett. 51(22), 1813-1815 (2015).

[8] Hoffmann, F., Ritchie, M., Fioranelli, F., Charlish, A. and Griffiths, H., "Micro-Doppler based detection and tracking of UAVs with multistatic radar," 2016 IEEE Radar Conf., 1-6, IEEE (2016).

[9] Rahman, S. and Robertson, D. A., "Radar micro-Doppler signatures of drones and birds at K-band and W-band," Sci. Rep. 8(1), 17396 (2018).

[10] Robertson, D. A., Brooker, G. M. and Beasley, P. D. L., "Very low-phase noise, coherent 94GHz radar for micro-Doppler and vibrometry studies," Proc. SPIE 9077, Radar Sens. Technol. XVIII 9077, K. I. Ranney and A. Doerry, Eds., 907719, International Society for Optics and Photonics (2014). 